cambridge.org/hyg

\section{Short Paper}

Cite this article: Weng $\mathrm{C}-\mathrm{H}$, Saal A, McGuire DC, Chan PA (2021). Persistently high SARSCoV-2 positivity rate and incidence for Hispanic/Latinos during state reopening in an urban setting: a retrospective cohort study. Epidemiology and Infection 149, e25, 1-4. https://doi.org/10.1017/S0950268821000133

Received: 13 October 2020

Revised: 31 December 2020

Accepted: 12 January 2021

\section{Key words:}

COVID-19; Hispanic; positivity rate; reopening; SARS-CoV-2; urban

Author for correspondence:

Chien-Hsiang Weng, E-mail:

chien-hsiang_weng@brown.edu

\title{
Persistently high SARS-CoV-2 positivity rate and incidence for Hispanic/Latinos during state reopening in an urban setting: a retrospective cohort study
}

\section{Chien-Hsiang Weng ${ }^{1,2}$ (D), Andrew Saal ${ }^{2}$, Daniel C. McGuire ${ }^{2}$ and Philip A. Chan ${ }^{1}$}

\footnotetext{
${ }^{1}$ Brown University Warren Alpert Medical School, Providence, Rhode Island 02903, USA and ${ }^{2}$ Providence Community Health Centers, Providence, Rhode Island 02905, USA
}

\begin{abstract}
Hispanic/Latino populations are disproportionately impacted by coronavirus disease 2019 (COVID-19) in the United States. The impact of state reopening on COVID-19 in this population after stay-at-home orders is unknown. We evaluated the incidence, prevalence and trends during reopening of severe acute respiratory syndrome-coronavirus-2 (SARS-CoV-2) at a major federally qualified health centre in Providence, Rhode Island. A total of 14505 patients were tested for SARS-CoV-2 from 19 March to 18 August 2020, of which, data on 13318 (91.8\%) patients were available; $70.0 \%$ were Hispanic/Latino, and 2905 were positive for SARS-CoV-2 infection. The urban Hispanic/Latino population was almost five times more likely to test positive for SARS-CoV-2 (risk ratio $4.97,95 \%$ CI 2.59-9.53, $P<0.001$ ) compared to nonHispanic White. The positivity rates among the urban Hispanic/Latino population remained $>10 \%$ during all phases of reopening. The trends of the incidence rates showed similar associations to those we observed for positivity rates. Public health interventions to address SARS-CoV2 in Hispanic/Latino communities are urgently needed, even in latter phases of state reopening.
\end{abstract}

Coronavirus disease 2019 (COVID-19), caused by severe acute respiratory syndrome coronavirus-2 (SARS-CoV-2), has resulted in a worldwide pandemic including over 19.8 million cases in the United States as of 31 December 2020 [1]. In the US, Hispanic/Latino populations are disproportionately impacted by SARS-CoV-2 [2, 3]. To address the pandemic, many states have implanted stay-at-home orders to limit the spread of the virus in the community [4]. As the pandemic has progressed, many states have gradually opened during several 'phases' which have included a loosening of restrictions on work and movement. Data on the impact of phased reopening on cases of SARS-CoV-2 in populations at higher-risk, including Hispanic/Latinos, are limited.

In the State of Rhode Island (RI), Hispanic/Latino individuals are up to three times more likely to test positive for SARS-CoV-2 [2]. The positivity rate of SARS-CoV-2 testing is an important measure in evaluating the spread and control of the disease. It is a relative approximation of how prevalent the virus is and is also dependent on the overall testing volume. States are using this to make policy decisions about quarantining visitors from other areas as well as to determine sufficient testing capacity. To address COVID-19 in Rhode Island, an initial stay-at-home order was implemented on 28 March 2020. Peak COVID-19 cases were observed in late April. Subsequently, the state underwent a series of phased re-opening stages according to the disease trend [5]. Phase 1 was to lift the stay-at-home order and allow social gatherings up to 10 people (9 May 2020), while phase 2 allowed up to 15 people (1 June 2020) and phase 3 up to 50 people. During each phase, different settings were allowed back to work including restaurants, public beaches and other settings.

To understand how reopening affected SARS-CoV-2 cases, with a focus on Hispanic/ Latinos, we reviewed the data from a major federally qualified health centre (FQHC) in Providence, Rhode Island, which consisted of 10 neighborhood clinics and approximately 60000 patients or one third of the total population in Providence. FQHCs are community health centres which receive federal funding to provide primary care services in underserved areas. Providence is the largest urban centre in the state and consistently the town/city with the greatest number of reported SARS-CoV-2 cases. We examined the number of COVID-19 cases and SARS-CoV-2 per cent positivity over time across different race/ethnicity populations and determined the overall positivity rate (number positive/number tested) and incidence rate (number positive/population covered) per week. The patient population at the FQHC is predominantly Hispanic/Latino and $90 \%$ of households are under $200 \%$ federal poverty level (FPL), which is a measure of income issued every year by the Department of Health and Human Services. Different FPLs are used to determine the eligibility for certain programmes 
Table 1. Patient characteristics and results of SARS-CoV-2 testing

\begin{tabular}{|c|c|c|c|c|c|}
\hline & \multicolumn{3}{|c|}{ SARS-CoV-2 tested individuals at PCHC $(N=13318)$} & \multicolumn{2}{|c|}{$\begin{array}{l}\text { SARS-CoV-2 tested individuals in } \\
\text { Rhode Island ( } N=224929)\end{array}$} \\
\hline & Positive & Negative & $P$ value & Positive & Negative \\
\hline Total - individual (\%) & $2905(21.8)$ & $10413(78.2)$ & & $19880(8.8)$ & $205049(91.2)$ \\
\hline \multicolumn{6}{|l|}{ Sex - no. (\%) } \\
\hline Female & $1830(63.0)$ & $6947(66.7)$ & $<0.001$ & $10963(55.1)$ & $110985(54.1)$ \\
\hline Male & $1075(37.0)$ & $3464(33.3)$ & & $8826(44.4)$ & $87300(42.6)$ \\
\hline Pending further information & 0 & $2(<1)$ & & $91(<1)$ & $6764(3.3)$ \\
\hline Median age - years (IQR) & $37.0(26.0-49.0)$ & $35.0(25.0-50.0)$ & 0.178 & - & - \\
\hline \multicolumn{6}{|l|}{ Race/Ethnicity - no. (\%) } \\
\hline Hispanic/Latino & $2382(82.0)$ & $6946(66.7)$ & $<0.001$ & $7274(36.6)$ & $12672(6.2)$ \\
\hline Non-Hispanic Black & $172(5.9)$ & $923(8.9)$ & & $1896(9.5)$ & $4987(2.4)$ \\
\hline Non-Hispanic White & $58(2.0)$ & $815(7.8)$ & & $5655(28.4)$ & $38177(18.6)$ \\
\hline Others (including race/ethnicity unknown) ${ }^{a}$ & $293(10.1)$ & $1729(16.6)$ & & $5055(25.4)$ & $149213(72.8)$ \\
\hline \multicolumn{6}{|l|}{ Age group (years old) - no. (\%) } \\
\hline $0-9$ & $138(4.7)$ & $620(6.0)$ & $<0.001$ & $604(3.0)$ & $6930(3.4)$ \\
\hline $10-19$ & $247(8.5)$ & $738(7.1)$ & & $1235(6.2)$ & $12028(5.9)$ \\
\hline 20-39 & $1194(41.1)$ & $4670(44.8)$ & & $6576(33.1)$ & $69482(33.9)$ \\
\hline $40-59$ & $1023(35.2)$ & $3104(29.8)$ & & $5955(30.0)$ & $60997(29.7)$ \\
\hline $60-79$ & $269(9.3)$ & $1141(11.0)$ & & $3477(17.5)$ & $42996(21.0)$ \\
\hline $80+$ & $34(1.2)$ & $140(1.3)$ & & $2028(10.2)$ & $12464(6.1)$ \\
\hline Pending further information & & & & 5 & 157 \\
\hline \multicolumn{6}{|l|}{ Insurance status - no. (\%) } \\
\hline Insured & $2250(77.5)$ & $9110(87.5)$ & $<0.001$ & - & - \\
\hline Uninsured & $655(22.5)$ & $1303(12.5)$ & & - & - \\
\hline
\end{tabular}

PCHC: Providence Community Health Centers; IQR: interquartile range.

${ }^{a}$ Others included: Asian, more than one race (non-Hispanic), native Hawaiian, other Pacific islander, race/ethnicity unknown.

and benefits. Every patient of this FQHC who was tested for SARS-CoV-2 by reverse-transcription polymerase chain reaction (RT-PCR) was included in this study from 19 March 2020. We characterised patients by demographics, insurance status and SARS-CoV-2 test results. If a patient had multiple positive tests, only the first test was counted for the purpose of this analysis. We reported numbers (percentages) for binary/categorical variables and medians (interquartile ranges, IQR) for continuous variables. Chi-square tests and Wilcoxon rank-sum tests were applied to determine statistically significant differences among groups. A two-sided significance threshold was set at $P<0.05$. The Providence Community Health Centers Review Committee approved the project. All analyses were run using STATA 13.1 (StataCorp, College Station, TX).

A total of 14505 patients were tested for SARS-CoV-2 from 19 March to 18 August 2020, of which, data on 13318 (91.8\%) patients were available; $65.9 \%$ were female, $70.0 \%$ were selfreported as Hispanic/Latino and $85.3 \%$ were insured. Overall, 11274 individuals had one test, 1871 had two tests and 173 had three or more (Table 1). Of the Hispanic/Latino patients tested, $25.5 \%(2832 / 9328)$ were positive, compared to $15.7 \%$ $(172 / 1095)$ of non-Hispanic (NH) Blacks, 6.6\% (58/873) of $\mathrm{NH}$ Whites and $14.5 \%(293 / 2022)$ of $\mathrm{NH}$ others $(P<0.001)$. A total of $82.0 \%$ of the total positive tests were from Hispanic/Latino patients, $6.1 \%$ from $\mathrm{NH}$ Black, $2.1 \%$ from $\mathrm{NH}$ White and $9.8 \%$ from $\mathrm{NH}$ others. The Hispanic/Latino population experienced a significant decrease in the positivity rates between phases 1 and $3(22.8-12.1 \%$, respectively). This was in comparison to the $\mathrm{NH}$ White population which experienced a $2.5 \%$ reduction and $\mathrm{NH}$ Blacks which experienced a $13.1 \%$ reduction. When comparing to statewide trends, Rhode Island experienced a $3.5 \%$ reduction in cases over the phased reopening with a steadily low positivity rate during phase 3 , similar to the $\mathrm{NH}$ White population at our clinic. Despite this, the positivity rate among the Hispanic/Latino population was still $>10 \%$ even during phase 3 (Fig. 1). During the entire period, the Hispanic/Latino population was almost five times more likely to test positive for SARS-CoV-2 (risk ratio 4.97, 95\% CI 2.59-9.53, $P<0.001$ ) compared to $\mathrm{NH}$ White, and 2.6 times more likely compared to NH Black (risk ratio $2.61,95 \%$ CI $1.70-4.00, P<0.001$ ). The subgroup analysis by their insurance status, which may reflect the socioeconomic or legal status, we found much higher positivity rates in all race/ethnicity groups who were uninsured except the $\mathrm{NH}$ Blacks. Uninsured Hispanic/Latino patients were still 2.6 and 2.4 times more likely to test positive for SARS-CoV-2 compared to the uninsured NH White and uninsured NH Black, respectively 


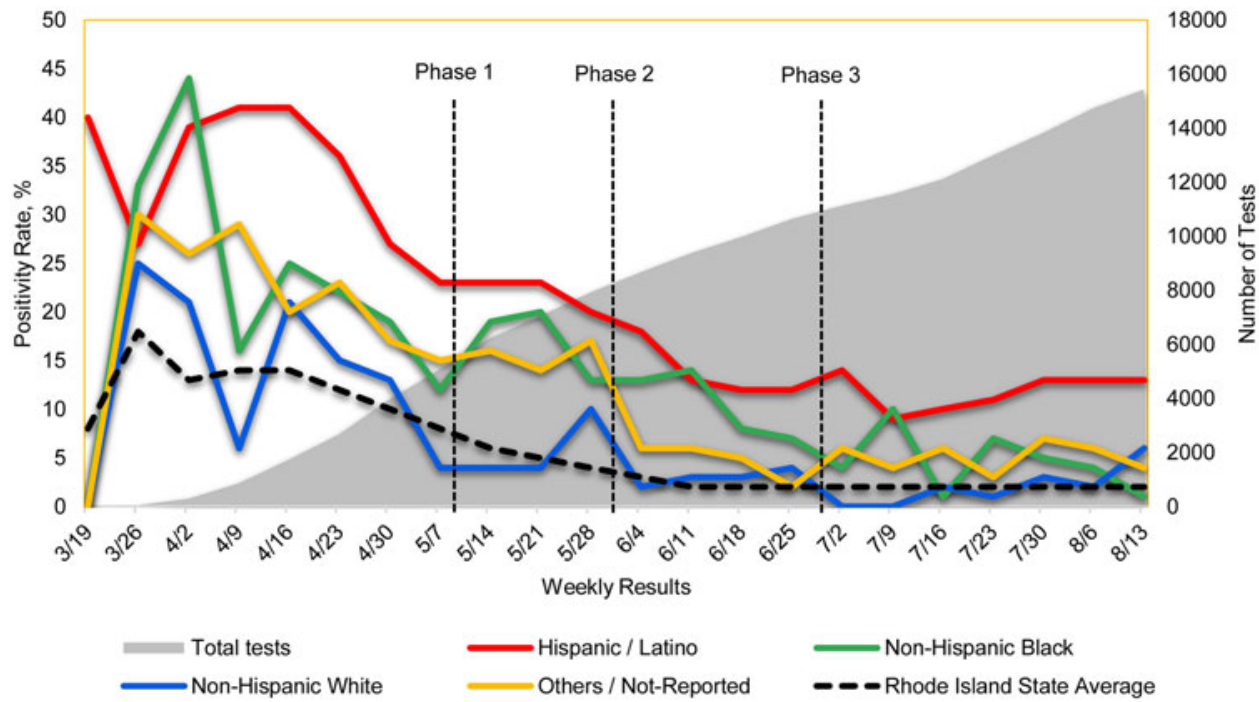

Fig. 1. SARS-CoV-2 positivity rates at Providence Community Health Centers vs. the Rhode Island State average. The red line represents the per cent positivity of the Hispanic/Latino population over reopening phases 1-3 compared to other populations. The per cent positivity of the Hispanic/Latino population never dropped below $10 \%$. The shaded grey area represents the total number of cumulative tests over time.

(risk ratio 2.59, 95\% CI 1.41-4.75, $P<0.001$; risk ratio $2.38,95 \%$ CI 1.55-3.66, $P<0.001)$. The positivity rate in NH Black population has dropped below $10 \%$ during phase 3. However, when compared to the $\mathrm{NH}$ White population, they were still two times more likely to test positive (risk ratio $2.11,95 \% \mathrm{CI}$ $1.59-2.81, P<0.001)$. A total of $21.2 \%$ Hispanic/Latino patients tested positive at the FQHC during the entire study period, which was significantly higher than the state average $(4.7 \%)$ as of 22 August 2020 [6]. Comparing to the statewide positivity rate, our Hispanic/Latino population was 4.5 times more likely to test positive for SARS-CoV-2 (risk ratio 4.51, 95\% CI 4.34-4.68, $P<0.001$ ), while NH Black population was 2.7 times (risk ratio $2.72,95 \%$ CI 2.36-3.13, $P<0.001$ ), and NH White was 1.29 times (95\% CI $1.00-1.65, P=0.048$ ).
We also compared the weekly incidence rates between our Hispanic/Latino, NH Black, NH White populations and the statewide population. According to the United States Census Bureau, there are 179883 residents in Providence, RI and 1059361 in the State of Rhode Island [7]. There are 42240 Hispanic/Latino patients, $4538 \mathrm{NH}$ Black and $3991 \mathrm{NH}$ White among the communities our FQHC serves. The weekly incidence rates among the Hispanic/Latino population have been more than three times higher than the statewide incidence rates for the majority of the time since the pandemic throughout the reopening phases. $\mathrm{NH}$ Black as well had significantly higher incidence until phase 3 reopening while $\mathrm{NH}$ White had similar rates throughout the entire period compared to the statewide trend (Fig. 2). The incidence trends were consistent with the trends of the positivity

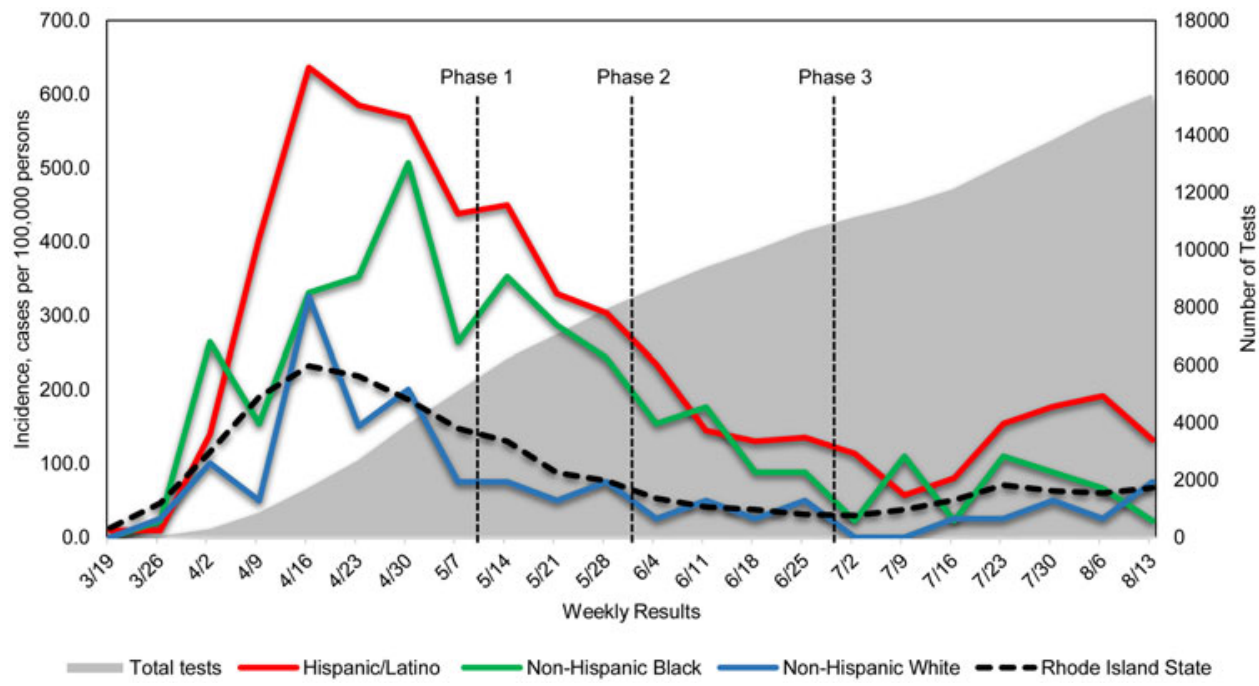

Fig. 2. New positive COVID-19 cases and estimated incidence by race/ethnicity group and week. The red line represents the weekly COVID-19 incidence of the Hispanic/Latino population over reopening phases 1-3 compared to other populations. The weekly incidence rates among the Hispanic/Latino population have been significantly higher than the statewide weekly incidence rates since the pandemic throughout the reopening phases. The shaded grey area represents the total number of cumulative tests over time. 
rates in our study. When comparing the incidence of COVID-19 to the statewide average, our Hispanic/Latino population was 3 times higher (incidence rate ratio 3.01, 95\% CI 2.88-3.14, $P<0.001$ ), while $\mathrm{NH}$ Black population was 2 times (incidence rate ratio $2.02,95 \% \mathrm{CI} 1.73-2.35, P<0.001)$ and $\mathrm{NH}$ White was slightly lower (incidence rate ratio $0.77,95 \%$ CI $0.59-1.00$, $P=0.045$ ).

This is among the first studies to evaluate changes in rates of SARS-CoV-2 among Hispanic/Latinos over time and during phased reopening, and demonstrates persistently high positivity rates and incidence in this population. Reassuringly, the urban Hispanic/Latino populations did experience a drop in cases over time as did other race/ethnicity groups. However, the positivity rates remained steadily above $10 \%$ in the Hispanic/Latino population in phase 3 without further reduction. We suspect that this might be similar in other urban areas. Positivity rates can be used as a marker of both access to testing, and burden of infection in the community. Positivity rates below $5 \%$ are generally considered adequate in terms of testing penetration and lower rates of infection [8]. The phased reopening was implemented based on the state-level incidence and positivity trends, which were inconsistent with those observed among the vulnerable population groups included in this study. The persistently higher rate $>10 \%$ in all phases of reopening suggest that more needs to be done in terms of access to SARS-CoV-2 testing, as well as focused interventions for this community.

The persistently high positive rate could be explained by several factors in the Hispanic/Latino population, including lack of access to health care and SARS-CoV-2 testing or medical insurance, less opportunity for social distancing with multigenerational or multi-family housing, the necessity of continuing to work in face-to-face settings where there is a high risk of transmission for financial or employment concerns, or higher disease prevalence [2, 9]. Disparities among Hispanic/Latinos persist for other diseases as well [10] and exacerbated by social determinants of health. Improved efforts to address structural barriers related to COVID-19 and healthcare access in general include accessible testing sites specifically in Hispanic/Latino communities, improved access to care, culturally competent messaging and healthcare settings, focused outreach and media and other interventions specifically adapted to this community. In this study, with a relatively smaller $\mathrm{NH}$ Black population, we also found the significantly higher rates of being tested positive among the $\mathrm{NH}$ Black population compared to the $\mathrm{NH}$ Whites, though the positivity rate in $\mathrm{NH}$ Black population has dropped below $10 \%$. This may be more significant in other larger urban centres where there may be a larger $\mathrm{NH}$ Black population.

Limitations of this study include that it was a single study site and may not represent other settings in the United States. The positivity rate may depend on how many tests are done during that specific period of time. Our analysis involved trends based on reopening in Rhode Island which may differ by state. Despite this, we found that despite overall state trends, Hispanic/Latino populations may experience persistently higher rates of infection over time.
In conclusion, tailoring the policy to meet the needs of the Hispanic/Latino communities, and reinforcing COVID-19 mitigation approaches such as wearing face masks, physical distancing, hand washing and other measures may help further reduce the spread of SARS-CoV-2 infection in this population.

Author contributions. CW and PC conceptualised the study. CW and DM had full access to all of the data in the study. CW conducted the analysis. CW, AS, DM and PC interpreted the results. CW and PC drafted the manuscript. All authors reviewed the paper for content and approved the final version.

Financial support. This research received no specific grant from any funding agency, commercial or not-for-profit sectors.

\section{Conflict of interest:. None.}

Ethical standards. The study was approved by the Providence Community Health Centers Review Committee. The authors assert that all procedures contributing to this study comply with the ethical standards of the relevant national and institutional committees on human experimentation and with the Helsinki Declaration of 1975, as revised in 2008.

Data. The data that support the findings of this study are available on request from the corresponding author, $\mathrm{C}-\mathrm{H}$ W. The data are not publicly available due to their containing information that could compromise the privacy of research participants.

\section{References}

1. Johns Hopkins University School of Medicine. COVID-19 dashboard by the Center for Systems Science and Engineering (CSSE) at Johns Hopkins University (JHU). Available at https://coronavirus.jhu.edu/. Accessed 31 December 2020.

2. Weng CH, Saal A and Chan PA (2020) High prevalence of SARS-CoV-2 infection among the uninsured Hispanic/Latino population: a retrospective cohort study. Journal of General Internal Medicine 14, 1-3.

3. Figueroa JF et al. (2020) Community-level factors associated with racial and ethnic disparities in COVID-19 rates in Massachusetts. Health Affairs (Millwood) 39(11), 1984-1992. doi: 101377hlthaff202001040.

4. Lurie MN et al. (2020) COVID-19 epidemic doubling time in the United States before and during stay-at-home restrictions. Journal of Infectious Diseases 222(10), 1601-1606.

5. State of Rhode Island and Providence Plantations Executive Order. Available at https://governor.ri.gov/documents/orders/Executive-Order20-14.pdf. Accessed 13 October 2020.

6. Rhode Island Department of Health COVID-19 Response Data. Available at https://ri-department-of-health-covid-19-data-rihealth.hub. arcgis.com/. Accessed 22 August 2020.

7. United States Census Bureau. QuickFacts Providence City, Rhode Island. Available at https://www.census.gov/quickfacts/fact/table/RI,providence countyrhodeisland,providencecityrhodeisland/PST045219. Accessed 13 October 2020.

8. Harvard Global Health Institute. New Testing Targets. Available at https://globalhealth.harvard.edu/new-testing-targets-as-covid-19-outbreaksgrow-more-severe-most-u-s-states-still-fall-far-short-on-testing/. Accessed 13 October 2020.

9. Martinez DA et al. (2020) SARS-CoV-2 positivity rate for latinos in the Baltimore-Washington, DC region. JAMA 324(4), 392-395.

10. Centers for Disease Control and Prevention. Health, United States Spotlight: Racial and Ethnic Disparities in Heart Disease. Available at https://www.cdc.gov/nchs/hus/spotlight/2019-heart-disease-disparities.htm. Accessed 13 October 2020. 